Article

\title{
Existence of Ulam Stability for Iterative Fractional Differential Equations Based on Fractional Entropy
}

\author{
Rabha W. Ibrahim ${ }^{1, *}$ and Hamid A. Jalab ${ }^{2}$ \\ ${ }^{1}$ Institute of Mathematical Sciences, University Malaya, Kuala Lumpur 50603, Malaysia \\ ${ }^{2}$ Faculty of Computer Science and Information Technology, University Malaya, Kuala Lumpur 50603, \\ Malaysia; E-Mail: hamidjalab@um.edu.my \\ * Author to whom correspondence should be addressed; E-Mail: rabhaibrahim@yahoo.com. \\ Academic Editors: J. A. Tenreiro Machado and António M. Lopes
}

Received: 12 March 2015 / Accepted: 11 May 2015 / Published: 13 May 2015

\begin{abstract}
In this study, we introduce conditions for the existence of solutions for an iterative functional differential equation of fractional order. We prove that the solutions of the above class of fractional differential equations are bounded by Tsallis entropy. The method depends on the concept of Hyers-Ulam stability. The arbitrary order is suggested in the sense of Riemann-Liouville calculus.
\end{abstract}

Keywords: fractional calculus; fractional differential equation; entropy solution

PACS classifications: 02.70

\section{Introduction}

In 1940, Ulam [1,2] established the following question on the stability of the Cauchy equation: if a function $\phi$ approximately satisfies functional equation $\mathcal{E}$, when does an exact solution of $\mathcal{E}$ which $\phi$ approximates exist?

In 1941, D. H. Hyers introduced a solution to the problem [3]. In 1978, Th.M. Rassias [4] modified Hyers' theorem by establishing the existence of unique linear mappings near approximate additive mappings. The issue has been assumed for various types of spaces (see [5-7]). Li and Hua [8] investigated the Hyers-Ulam stability of finite polynomial equations. Bidkham, Mezerji, and Gordji [9] introduced the Hyers-Ulam stability of the generalized finite polynomial equation. Recently, Cimpean and Popa [10] proved the Hyers-Ulam stability for Euler's equation in $\mathbb{R}^{n}$. Popa and Rasa [11] obtained 
the infimum of the Hyers-Ulam stability constants for different operators, such as the Stancu, Bernstein, and Kantorovich operators.

The Ulam stability of fractional differential equations was introduced for the first time by Wang and Zhou using the Caputo derivative [12]. Ibrahim investigated this type of stability for different classes of fractional differential equations by utilizing the fractional derivatives of the Srivastava-Owa differential operator (a type of Riemann-Liouville operator) in a complex domain [13-15]. The Ulam-Hyers stability for the Cauchy fractional differential equation in the unit disk was also investigated. Various studies are reported in [16,17]. Wang and Lin also imposed stability by utilizing the Hadamard-type fractional integral equations [18]. Recently, the stability of the sequential fractional differential equation with respect to the Miller-Ross formula was investigated on the basis of the Banach contraction mapping theorem [19].

The class of fractional iterative differential equations is derived by applying non-expansive operators $[20,21]$ as follows:

$$
D^{\rho} y(t)=\phi\left(t, y(t), y((y(t))), \quad y(0)=y_{0}\right.
$$

Ibrahim and Darus [22] established sufficient conditions for fractional differential equations as follows:

$$
D^{\rho} y(t)=\phi(t, y(t), y(\beta t), y(y(t))), \quad \beta \in(0,1]
$$

with the initial value $y(0)=y_{0}$. Recently, Ibrahim et al. [23] investigated the existence and uniqueness of

$$
D^{\rho} y(t)=\phi\left(t, y^{[1]}(t), y^{[2]}(t), \ldots, y^{[n]}(t)\right)
$$

subjected to the initial value as follows:

$$
y\left(t_{0}\right)=c, \quad c \in[0, \infty)
$$

where $y^{[j]}(t):=y\left(y^{[j-1]}(t)\right)$ indicates the $j-t h$ iterate of self-mapping $y$, where $j=1,2, \ldots, n$.

In this study, we impose conditions for the existence of solutions for an iterative functional differential equation of fractional order Equation (1). We prove that the solutions of the aforementioned class of fractional differential equations are bounded by the Tsallis entropy (fractional entropy). The strategy is based on the concept of the Hyers-Ulam stability. The arbitrary order is derived with respect to the Riemann-Liouville calculus.

\section{Preliminaries}

We consider the following concepts:

In view of the Riemann-Liouville operators (differential and integral), the fractional calculus can be defined and the fractional integral operator can be formulated as follows:

$$
I_{a}^{\rho} \phi(s)=\int_{a}^{s} \frac{(s-\tau)^{\rho-1}}{\Gamma(\rho)} \phi(\tau) d \tau
$$

Moreover, for the continuous function $\phi$, the fractional derivative of order $\rho>0$ is defined by

$$
D_{a}^{\rho} \phi(s)=\frac{d}{d s} \int_{a}^{s} \frac{(s-\tau)^{-\rho}}{\Gamma(1-\rho)} \phi(\tau) d \tau
$$


Consequently, when $a=0$ we have

$$
D^{\rho} s^{m}=\frac{\Gamma(m+1)}{\Gamma(m-\rho+1)} s^{m-\rho}, m>-1 ; 0<\rho<1
$$

and

$$
I^{\rho} s^{m}=\frac{\Gamma(m+1)}{\Gamma(m+\rho+1)} s^{m+\rho}, m>-1 ; \rho>0
$$

Definition 1. The function $\phi: \mathbb{R}_{+}^{n} \rightarrow \chi, n \in \mathbb{N}$ is called homogeneous of degree $\gamma$ with respect to $\lambda \in \mathbb{R}_{+}$such that

$$
\begin{aligned}
\phi\left(\lambda y^{[1]}(t), \lambda y^{[2]}(t), \ldots, \lambda y^{[n]}(t)\right) & =\lambda^{\gamma} \phi\left(y^{[1]}(t), \ldots, y^{[n]}(t)\right) \\
& :=\lambda^{\gamma} \phi\left(y_{1}(t), \ldots, y_{n}(t)\right)
\end{aligned}
$$

where $\mathbb{R}_{+}=(0, \infty)$, and $(\chi,\|\|$.$) is a Banach space over \mathbb{R}$.

Definition 2. The function $\phi: \mathbb{R}_{+}^{n+1} \rightarrow \chi, n \in \mathbb{N}$ is called homogeneous of degree $0<\gamma \leq 1$ with respect to $t \in \mathbb{R}_{+}$if

$$
\begin{aligned}
\phi\left(t, t y^{[1]}(t), t y^{[2]}(t), \ldots, t y^{[n]}(t)\right) & =t^{\gamma} \phi\left(y^{[0]}(t), y^{[1]}(t), \ldots, y^{[n]}(t)\right) \\
& :=t^{\gamma} \phi\left(y_{0}(t), y_{1}(t), \ldots, y_{n}(t)\right)
\end{aligned}
$$

where $y_{0}(t)=y^{[0]}(t):=t^{\beta}, 0<\beta \leq 1$.

Definition 3. Let $\epsilon$ be a nonnegative number. Then, Equation (1) is considered stable in the Hyers-Ulam sense if $\delta>0$ such that for every $\phi \in C^{1} \in\left(\mathbb{R}_{+}^{n+1}, \chi\right)$, we derive Equation (2) and

$$
\left\|D^{\rho} y(t)-\phi\left(y_{0}(t), y_{1}(t), \ldots, y_{n}(t)\right)\right\| \leq \epsilon
$$

for all $y \in \mathbb{R}_{+}$the function $\eta \in \mathbb{R}_{+}$exists with the following property:

$$
\|y(t)-\eta(t)\| \leq \delta
$$

An entropy of the scalar variable was imposed by Mathai, and the properties and its links to the Tsallis non-extensive statistical mechanics and the Mathai pathway pattern were introduced and generalized by Mathai and Haubold [24]. In the present work, we deal with the following measure of entropy, which was derived by Tsallis [25]

$$
\mathcal{T}_{\gamma}(\phi)=\frac{\int_{x}[\phi(x)]^{\gamma} d x-1}{1-\gamma}, \quad \gamma \neq 1
$$

The discreet form is as follows:

$$
\mathcal{T}_{\gamma}(\phi)=\frac{1}{\gamma-1}\left(1-\sum_{i=1}^{m} \phi_{i}^{\gamma}\right)
$$

Various fractional entropies have been proposed in the literature [26-30]. We show that a solution of Equation (1) is bounded by the Tsallis entropy under some conditions. This solution satisfies the Hyers-Ulam stability. 


\section{Main results}

Our first main result is explained in the following theorem:

Theorem 1. Suppose that $\phi \in C\left(\mathbb{R}_{+}^{n+1}, \chi\right)$ achieving (2) and

$$
\|\phi\| \leq \frac{\Gamma(\rho)}{(1-\gamma) T^{\rho-1}}, \quad \gamma \in(0,1), t \in(0, T], \rho \in(0,1]
$$

If $c>\frac{1}{\gamma}$ then every solution y of Equation (1) is bounded by the Tsallis entropy.

Proof. The relationship

$$
\phi\left(t, t y^{[1]}(t), t y^{[2]}(t), \ldots, t y^{[n]}(t)\right)=t^{\gamma} \phi\left(y_{0}(t), y_{1}(t), \ldots, y_{n}(t)\right)
$$

implies that

$$
\phi\left(y_{0}(t), y_{1}(t), \ldots, y_{n}(t)\right)=\frac{\phi\left(t, t y^{[1]}(t), t y^{[2]}(t), \ldots, t y^{[n]}(t)\right)}{t^{\gamma}}
$$

By letting $t=\frac{1}{y_{0}}$ we derive the following equation:

$$
\phi\left(y_{0}(t), y_{1}(t), \ldots, y_{n}(t)\right)=y_{0}^{\gamma} \phi\left(1, \frac{y_{1}(t)}{y_{0}(t)}, \ldots, \frac{y_{n}(t)}{y_{0}(t)}\right)
$$

Let $y$ be a solution of the form

$$
y(t)=c+\int_{0}^{t} \frac{(t-\tau)^{\rho-1}}{\Gamma(\rho)} \phi\left(\tau, y_{1}(\tau), \ldots, y_{n}(\tau)\right) d \tau
$$

Then in view of Equation (5) yields

$$
\begin{aligned}
y(t) & =c+\int_{0}^{t} \frac{(t-\tau)^{\rho-1}}{\Gamma(\rho)} \phi\left(\tau, y_{1}(\tau), \ldots, y_{n}(\tau)\right) d \tau \\
& \leq c+\int_{0}^{t} y_{0}^{\gamma}(\tau)\|\phi\| \frac{(t-\tau)^{\rho-1}}{\Gamma(\rho)} d \tau \\
& \leq c+\frac{\int_{0}^{t} y_{0}^{\gamma}(\tau) d \tau}{1-\gamma} \\
& =\frac{c-c \gamma}{1-\gamma}+\frac{\int_{0}^{t} y_{0}^{\gamma}(\tau) d \tau}{1-\gamma}
\end{aligned}
$$

If $c>\frac{1}{\gamma}$ then we obtain the following inequality:

$$
y(t) \leq \frac{c}{1-\gamma}+\frac{\int_{0}^{T} y_{0}^{\gamma}(t) d t-1}{1-\gamma}
$$

Stability is discussed in the subsequent result. 
Theorem 2. Assume that $\phi \in C\left(\mathbb{R}_{+}^{n+1}, \chi\right)$ achieving (2) and

$$
\|\phi\| \leq \frac{\Gamma(\rho)}{(1-\gamma) T^{\rho-1}}, \quad \gamma \in(0,1), t \in(0, T], \rho \in(0,1]
$$

If $c>\frac{1}{\gamma}$, then a unique solution y of Equation (1) satisfies the Hyers-Ulam stability.

Proof. We let $y$ be a solution of Equation (1) satisfying Equation (3). Putting the following equation:

$$
D^{\rho} y(t)-\phi\left(y_{0}(t), y_{1}(t), \ldots, y_{n}(t)\right):=\Phi(t)
$$

If $\|\Phi\| \leq \epsilon, \epsilon>0$, then

$$
\int_{0}^{t} \frac{(t-\tau)^{\rho-1}}{\Gamma(\rho)} \Phi(\tau) d \tau, \quad t \rightarrow T
$$

is absolutely convergent. We consider the following function:

$$
\eta(t)=\frac{\int_{0}^{T} y_{0}^{\gamma}(t) d t-1}{1-\gamma}+\int_{0}^{t} \frac{(t-\tau)^{\rho-1}}{\Gamma(\rho)} \Phi(\tau) d \tau+\frac{c}{1-\gamma}
$$

Thus, in view of Theorem 1, we derive the following equation:

$$
y(t) \leq \frac{c}{1-\gamma}+\frac{\int_{0}^{T} y_{0}^{\gamma}(t) d t-1}{1-\gamma}
$$

Consequently, we obtain the following inequality:

$$
\begin{aligned}
\|y(t)-\eta(t)\| & =\left\|y(t)-\left(\frac{\int_{0}^{T} y_{0}^{\gamma}(t) d t-1}{1-\gamma}+\int_{0}^{t} \frac{(t-\tau)^{\rho-1}}{\Gamma(\rho)} \Phi(\tau) d \tau+\frac{c}{1-\gamma}\right)\right\| \\
& \leq\left\|\frac{c}{1-\gamma}+\frac{\int_{0}^{T} y_{0}^{\gamma}(t) d t-1}{1-\gamma}-\left(\frac{\int_{0}^{T} y_{0}^{\gamma}(t) d t-1}{1-\gamma}+\int_{0}^{t} \frac{(t-\tau)^{\rho-1}}{\Gamma(\rho)} \Phi(\tau) d \tau+\frac{c}{1-\gamma}\right)\right\| \\
& =\left\|\int_{0}^{t} \frac{(t-\tau)^{\rho-1}}{\Gamma(\rho)} \Phi(\tau) d \tau\right\| \\
& \leq \int_{0}^{t}\left\|\frac{(t-\tau)^{\rho-1}}{\Gamma(\rho)} \Phi(\tau)\right\| d \tau \\
& \leq\|\Phi\| \int_{0}^{t} \frac{(t-\tau)^{\rho-1}}{\Gamma(\rho)} d \tau, \quad t>\tau \\
& \leq \frac{\epsilon T^{\rho}}{\Gamma(\rho+1)}:=\delta, \quad\|\Phi\|<\epsilon
\end{aligned}
$$

The existence of stability is proven. As such, we assume two solutions to Equation (1), namely, $y$ and $u$ such that $y \neq u$ with the property in Equation (6). Thus, there exists $t^{0} \in(0, T]$ such that

$$
y\left(t^{0}\right) \neq u\left(t^{0}\right)
$$

For all $t>0$ the following inequality holds:

$$
\begin{aligned}
\left\|y\left(t^{0}\right)-u\left(t^{0}\right)\right\| & \leq\left\|y\left(t^{0}\right)-\eta\left(t^{0}\right)\right\|+\left\|\eta\left(t^{0}\right)-u\left(t^{0}\right)\right\| \\
& \leq \frac{2 \epsilon T^{\rho}}{\Gamma(\rho+1)}
\end{aligned}
$$

Since $\epsilon$ is arbitrary, which leads to a contradiction. Thus, we prove the uniqueness of the solution. 
Next, we discuss the stability of the following case:

$$
D^{\rho} y(t)=\sum_{i=0}^{m} A_{i}(t) y^{[i]}(t)
$$

subjected to the initial value

$$
y\left(t_{0}\right)=c, \quad c \in[0, \infty), \quad t_{0}>0
$$

We formulate the following theorem:

Theorem 3. Assume that

$$
F(y):=D^{\rho} y(t)-\sum_{i=0}^{m} A_{i}(t) y^{[i]}(t), \quad t \in(0, T]
$$

such that $|F(u)|<\epsilon, \epsilon>0$, and

$$
|F(y)-F(v)| \leq \lambda|y-v|
$$

for some $\lambda<\frac{1-\gamma}{2 \Gamma(\rho) T^{2-\rho}}$. If

$$
\sum_{i=0}^{\infty} \max _{t}\left|A_{i}(t)\right| \leq \sum_{i=0}^{\infty} \frac{\Gamma(\rho)}{(1-\gamma) 2^{i} T^{\rho-1}}, \quad \gamma \in[0,1)
$$

then Equation (7) has Hyers-Ulam stability.

Proof. The solution to Equation (7) has the following form:

$$
\begin{aligned}
y(t) & =c+\int_{0}^{t} \frac{(t-\tau)^{\rho-1}}{\Gamma(\rho)} \sum_{i=0}^{m} A_{i}(\tau) y^{[i]}(\tau) d \tau \\
& =c+\sum_{i=0}^{m} \int_{0}^{t} \frac{(t-\tau)^{\rho-1}}{\Gamma(\rho)} A_{i}(\tau) y^{[i]}(\tau) d \tau \\
& =c+\sum_{i=0}^{m} \int_{0}^{t} \frac{(t-\tau)^{\rho-1}}{\Gamma(\rho)} A_{i}(\tau) y_{0}^{\gamma} y_{i}(\tau) d \tau
\end{aligned}
$$

Moreover, we determine that

$$
\begin{aligned}
y(t) & =\int_{0}^{t} \frac{(t-\tau)^{\rho-1}}{\Gamma(\rho)} F(\tau) d \tau+\sum_{i=0}^{m} \int_{0}^{t} \frac{(t-\tau)^{\rho-1}}{\Gamma(\rho)} A_{i}(\tau) y_{0}^{\gamma} y_{i}(\tau) d \tau \\
& \leq \frac{\epsilon T^{\rho}}{\Gamma(\rho+1)}+\int_{0}^{t} y_{0}^{\gamma} \frac{(t-\tau)^{\rho-1}}{\Gamma(\rho)} \sum_{i=0}^{m} A_{i}(\tau) y_{i}(\tau) d \tau \\
& \leq \frac{\epsilon T^{\rho}}{\Gamma(\rho+1)}+2|y(t)| \int_{0}^{t} \frac{y_{0}^{\gamma}}{1-\gamma} d \tau \\
& =\frac{\epsilon T^{\rho}}{\Gamma(\rho+1)}+2|y(t)| \frac{\int_{0}^{T} y_{0}^{\gamma} d t-1}{1-\gamma}+\frac{2|y(t)|}{1-\gamma} \\
& =\frac{\epsilon T^{\rho}}{\Gamma(\rho+1)}+2|y(t)| \mathcal{T}_{\gamma}\left(y_{0}\right)+\frac{2|y(t)|}{1-\gamma}
\end{aligned}
$$


where $|y(t)|=\max y_{i}(t)$. Thus, we obtain the following inequality:

$$
|y(t)| \leq \frac{\frac{\epsilon T^{\rho}}{\Gamma(\rho+1)}}{1-2\left(\mathcal{T}_{\gamma}\left(y_{0}\right)+\frac{1}{1-\gamma}\right)}:=K_{\epsilon}
$$

We define the function $G: L_{1}(0, T] \longrightarrow L_{1}(0, T]$ as follows:

$$
G(y)=\sum_{i=0}^{m} A_{i}(t) F(y)
$$

Evidently, $G(y)$ is absolutely convergent because

$$
\begin{aligned}
\lim _{m \rightarrow \infty}|G(y)| & =\mid\left(\sum_{i=0}^{\infty} A_{i}(t) F(y) \mid\right. \\
& \leq \sum_{i=0}^{\infty} \max _{t}\left|A_{i}(t)\right||F(y)| \\
& \leq \sum_{i=0}^{\infty} \frac{\Gamma(\rho)|F(y)|}{2^{j}(1-\gamma) T^{\rho-1}} \\
& \leq \frac{2 \Gamma(\rho) \epsilon}{(1-\gamma) T^{\rho-1}}
\end{aligned}
$$

Moreover, $G(y)$ satisfies the following inequality:

$$
\begin{aligned}
|G(y)-G(v)| & =\left|\left(\sum_{i=0}^{m} A_{i}(t)\right) F(y)-\left(\sum_{i=0}^{m} A_{i}(t)\right) F(v)\right| \\
& \leq\left|\left(\sum_{i=0}^{m} A_{i}(t)\right)\right||F(y)-F(v)| \\
& \leq\left|\left(\sum_{i=0}^{m} A_{i}(t)\right)\right| \lambda|y-v|
\end{aligned}
$$

Thus for $m \rightarrow \infty$ we derive the following conclusion:

$$
\begin{aligned}
|G(y)-G(v)| & \leq \sum_{i=0}^{\infty} \frac{\lambda\|u-v\|}{2^{i}} \\
& =\frac{2 \Gamma(\rho) \lambda}{(1-\gamma) T^{\rho-1}}\|u-v\|
\end{aligned}
$$

Hence we conclude that

$$
\begin{aligned}
\|G(y)-G(v)\|_{L_{1}(0, T]} & =\int_{0}^{T}|G(y)-G(v)| d t \\
& \leq \frac{2 \Gamma(\rho) \lambda T^{2-\rho}}{(1-\gamma)}\|y-v\| \\
& :=\mu\|y-v\|, \quad \mu<1
\end{aligned}
$$

Therefore, $G$ is a $\mu$-contraction mapping in $L_{1}(0, T]$. On the basis of the Banach contraction mapping theorem, we find a unique $v \in L_{1}(0, T]$ such that

$$
G(v)=v .
$$


Then, we verify the stability of Equation (7). For $m \rightarrow \infty$ we get

$$
\begin{aligned}
|y(t)-v(t)| & =|y(t)-G(y)+G(y)-G(v)| \\
& \leq|y(t)-G(y)|+|G(y)-G(v)| \\
& \left.\left.\leq\left|y(t)+\sum_{i=0}^{m} A_{i}(t) F(y)\right|+\mid \sum_{i=0}^{m} A_{i}(t)\right) F(y)-\sum_{i=0}^{m} A_{i}(t)\right) F(v) \mid \\
& \leq|y(t)|\left(1+\sum_{i=0}^{\infty} A_{i}(t) \lambda\right)+\sum_{i=0}^{\infty} A_{i}(t) \lambda|y(t)-v(t)| \\
& \leq K_{\epsilon}\left(1+\frac{2 \lambda \Gamma(\rho)}{(1-\gamma) T^{\rho-1}}\right)+\left(\frac{2 \lambda \Gamma(\rho)}{(1-\gamma) T^{\rho-1}}\right)|y(t)-v(t)|
\end{aligned}
$$

Thus, we propose that

$$
|y(t)-v(t)| \leq K_{\epsilon}\left(\frac{1+\Lambda}{1-\Lambda}\right):=\delta, \quad \Lambda:=\frac{2 \lambda \Gamma(\rho)}{(1-\gamma) T^{\rho-1}}
$$

Therefore, Equation (7) has Ulam-Hyers stability.

\section{Conclusions}

In this study, we investigated the generalized Ulam-Hyers stability and Ulam-Hyers-Rassias stability for iterative fractional differential equations with respect to the Riemann-Liouville derivative. These stabilities depend on fractional entropy. We demonstrated that the solutions of the aforementioned class of fractional differential equations are bounded by the Tsallis entropy. We also utilized the Banach contraction fixed-point theorem.

\section{Acknowledgments}

The authors are grateful to the referees for their helpful suggestions that improved this article. This research is supported by Project No. RG312-14AFR from the University of Malaya.

\section{Author Contributions}

Both authors jointly worked on deriving the results and approved the final manuscript.

\section{Conflicts of Interest}

The authors declare no conflict of interest.

\section{References}

1. Ulam, S.M. A Collection of Mathematical Problems; Interscience Publishers: New York, NY, USA, 1960.

2. Ulam, S.M Problems in Modern Mathematics; Wiley: New York, NY, USA, 1964.

3. Hyers, D.H. On the stability of linear functional equation. Proc. Natl. Acad. Sci. USA 1941, 27, 222-224.

4. Rassias, T.M. On the stability of the linear mapping in Banach space. Proc. Am. Math. Soc. 1978, 72, 297-300. 
5. Hyers, D.H. The stability of homomorphisms and related topics. In GlobalAnalysts-Analysis on Mamfolds; Rassias, Th.M., Ed.; Springer: Berlin/Heidelberg, Germany, 1983; pp. 140-153.

6. Hyers, D.H., Rassias, Th.M. Approximate homomorphisms. Aequ. Math. 1992, 44, 125-153.

7. Hyers, D.H.; Isac G.I.; Rassias, T.M. Stability of Functional Equations in Several Variables; Birkhauser: Basel, Switzerland, 1998.

8. Li, Y.; Hua, L. Hyers-Ulam stability of a polynomial equation. Banach J. Math. Anal. 2009, 3, 86-90.

9. Bidkham, M.; Mezerji, H.A.; Gordji, M.E. Hyers-Ulam stability of polynomial equations. Abstr. Appl. Anal. 2010, 2010, doi:10.1155/2010/754120.

10. Cimpean, D.; Popa, D. Hyers-Ulam stability of Euler's equation. Appl. Math. Lett. 2011, 24, 1539-1543.

11. Popa, D.; Rasa, I. On the best constant in Hyers-Ulam stability of some positive linear operators. J. Math. Anal. Appl. 2014, 412, 103-108.

12. Wang, J.; Lv, L.; Zhou, Y. Ulam stability and data dependence for fractional differential equations with Caputo derivative. Electron. J. Qual. Theory Differ. Equ. 2011, 63, Available online: http://www.emis.ams.org/journals/EJQTDE/p1003.pdf (accessed on 12 May 2015).

13. Ibrahim, R.W. Approximate solutions for fractional differential equation in the unit disk. Electron. J. Qual. Theory Differ. Equ. 2011, 64, Available online: http://www.emis.ams.org/journals/EJQTDE/p603.pdf (accessed on 12 May 2015).

14. Ibrahim, R.W. Ulam stability for fractional differential equation in complex domain. Abstr. Appl. Anal. 2012, 2012, doi:10.1155/2012/649517.

15. Ibrahim, R.W. Generalized Ulam-Hyers stability for fractional differential equations. Int. J. Math. 2012, 23, 1250056.

16. Wang, J.; Zhou, Y.; Feckan, M. Nonlinear impulsive problems for fractional differential equations and Ulam stability. Comput. Math. Appl. 2012, 64, 3389-3405.

17. Wang, J.; Feckan, M.; Zhou, Y. Ulam's type stability of impulsive ordinary differential equations. J. Math. Anal. Appl. 2012, 395, 258-264.

18. Wang, J.; Lin, Z. Ulam's type stability of Hadamard type fractional integral equations. Filomat 2014, 28, 1323-1331.

19. Ibrahim, R.W. Stability of sequential fractional differential equation. Appl. Comput. Math. 2015, in press.

20. Ibrahim, R.W. Existence of deviating fractional differential equation. CUBO Math. J. 2012, 14, 129-142.

21. Ibrahim, R.W. Existence of iterative Cauchy fractional differential equation. J. Math. 2013, 2013, doi:10.1155/2013/838230.

22. Ibrahim, R.W.; Darus, M. Infective disease processes based on fractional differential equation. In Proceedings of the 3rd International Conference on Mathematical Sciences, Kuala Lumpur, Malaysia, 17-19 December 2013; Volume 1602, pp. 696-703.

23. Ibrahim, R.W.; Kilicman, A.; Damag, F. H. Existence and uniqueness for a class of iterative fractional differential equations. Adv. Differ. Equ. 2015, 421, doi:10.1186/s13662-015-0421-y.

24. Mathai, A.; Haubold, H. On a generalized entropy measure leading to the pathway model with a preliminary application to solar neutrino data. Entropy 2013, 15, 4011-4025.

25. Tsallis, C. Introduction to Nonextensive Statistical Mechanics, Approching a Complex World; Springer: New York, NY, USA, 2009.

26. Machado, J.A.T. Entropy analysis of integer and fractional dynamical systems. J. Appl. Nonlinear Dyn. 2010, 62, 371-378.

27. Machado, J.A.T. Entropy analysis of fractional derivatives and their approximation. J. Appl. Nonlinear Dyn. 2012, $1,109-112$. 
28. Ibrahim, R.W. The fractional differential polynomial neural network for approximation of functions. Entropy 2013, 15, 4188-4198.

29. Machado, J.A.T. Fractional order generalized information. Entropy 2014, 16, 2350-2361.

30. Ibrahim, R.W.; Jalab, H.A. Existence of entropy solutions for nonsymmetric fractional systems. Entropy 2014, 16, 4911-4922.

(c) 2015 by the authors; licensee MDPI, Basel, Switzerland. This article is an open access article distributed under the terms and conditions of the Creative Commons Attribution license (http://creativecommons.org/licenses/by/4.0/). 